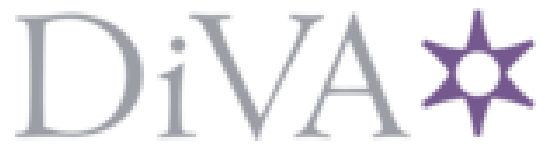

http://www.diva-portal.org

This is the published version of a paper published in Applied Physics Letters.

Citation for the original published paper (version of record):

Shi, C., Sun, N., Wu, Z., Chen, J., Ahamad, T. et al. (2018)

Managing excitons for high performance hybrid white organic light-emitting diodes by using a simple planar heterojunction interlayer.

Applied Physics Letters, 112(2): 023301

https://doi.org/10.1063/1.5000894

Access to the published version may require subscription.

N.B. When citing this work, cite the original published paper.

Permanent link to this version:

http://urn.kb.se/resolve?urn=urn:nbn:se:umu:diva-144351 


\section{Managing excitons for high performance hybrid white organic light-emitting diodes by using a simple planar heterojunction interlayer}

Changsheng Shi, Ning Sun, Zhongbin Wu, Jiangshan Chen, Tansir Ahamad, Saad M. Alshehri, and Dongge Ma

Citation: Appl. Phys. Lett. 112, 023301 (2018);

View online: https://doi.org/10.1063/1.5000894

View Table of Contents: http://aip.scitation.org/toc/apl/112/2

Published by the American Institute of Physics

\section{Articles you may be interested in}

Polarization and charge-transfer effect on the transport properties in two-dimensional electron gases $/ \mathrm{LaNiO}_{3}$ heterostructure

Applied Physics Letters 112, 021601 (2018); 10.1063/1.5013070

Low voltage operation of IGZO thin film transistors enabled by ultrathin $\mathrm{Al}_{2} \mathrm{O}_{3}$ gate dielectric

Applied Physics Letters 112, 023501 (2018); 10.1063/1.5003662

Lithium ion intercalation in thin crystals of hexagonal TaSe 2 gated by a polymer electrolyte

Applied Physics Letters 112, 023502 (2018); 10.1063/1.5008623

Chiroptic response of ferroelectric liquid crystals triggered with localized surface plasmon resonance of achiral gold nanorods

Applied Physics Letters 112, 021102 (2018); 10.1063/1.5005054

Depletion-mode quantum dots in intrinsic silicon

Applied Physics Letters 112, 023102 (2018); 10.1063/1.5002646

Modulated spin orbit torque in a $\mathrm{Pt} / \mathrm{Co} / \mathrm{Pt} / \mathrm{YIG}$ multilayer by nonequilibrium proximity effect Applied Physics Letters 112, 022402 (2018); 10.1063/1.5006115

\section{Scilight}

Sharp, quick summaries illuminating the latest physics research

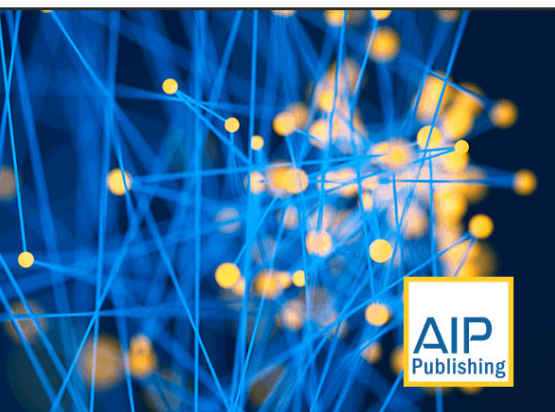




\title{
Managing excitons for high performance hybrid white organic light-emitting diodes by using a simple planar heterojunction interlayer
}

\author{
Changsheng Shi, ${ }^{1}$ Ning Sun, ${ }^{2}$ Zhongbin Wu, ${ }^{1}$ Jiangshan Chen, ${ }^{3}$ Tansir Ahamad, ${ }^{4}$ \\ Saad M. Alshehri, ${ }^{4}$ and Dongge $\mathrm{Ma}^{\uparrow, 3,4, a)}$ \\ ${ }^{1}$ State Key Laboratory of Polymer Physics and Chemistry, Changchun Institute of Applied Chemistry, \\ University of Chinese Academy of Sciences, Changchun 130022, People's Republic of China \\ ${ }^{2}$ Organic Photonics and Electronics Group, Department of Physics, Umea University, Umea SE-90187, \\ Sweden \\ ${ }^{3}$ State Key Laboratory of Luminescent Materials and Devices, Institute of Polymer Optoelectronic Materials \\ and Devices, South China University of Technology, Guangzhou 510640, People's Republic of China \\ ${ }^{4}$ Department of Chemistry, College of Science, King Saud University, Riyadh 11451, Kingdom of Saudi Arabia
}

(Received 19 August 2017; accepted 23 December 2017; published online 8 January 2018)

\begin{abstract}
High performance hybrid white organic light-emitting diodes (WOLEDs) were fabricated by inserting a planar heterojunction interlayer between the fluorescent and phosphorescent emitting layers (EMLs). The maximum external quantum efficiency (EQE) of $19.3 \%$, current efficiency of $57.1 \mathrm{~cd} \mathrm{~A}^{-1}$, and power efficiency (PE) of $66.2 \mathrm{~lm} \mathrm{~W}^{-1}$ were achieved in the optimized device without any light extraction enhancement. At the luminance of $1000 \mathrm{~cd} \mathrm{~m}^{-2}$, the EQE and PE remained as high as $18.9 \%$ and $60 \mathrm{~lm} \mathrm{~W}^{-1}$, respectively, showing the reduced efficiency-roll. In order to disclose the reason for such high performance, the distribution of excitons was analyzed by using ultra-thin fluorescent and phosphorescent layers as sensors. It was found that the heterojunction interlayer can efficiently separate the singlet and triplet excitons, preventing the triplet excitons from being quenched by the fluorescent emitter. The introduction of the heterojunction interlayer between the fluorescent and phosphorescent EMLs should offer a simple and efficient route to fabricate the high performance hybrid WOLEDs. Published by AIP Publishing.

https://doi.org/10.1063/1.5000894
\end{abstract}

Organic light-emitting diodes (OLEDs) are considered to be promising candidates for commercial lighting and display applications owing to their numerous advantages such as a long lifetime, high efficiency, and simple preparation. ${ }^{1-10}$ In a standard hybrid white organic light-emitting diode (WOLED), the singlet and triplet excitons can be used by fluorescent and phosphorescent materials, respectively, which can theoretically achieve 100\% internal quantum efficiency (IQE). However, a blue fluorescent emitting layer (EML) needs to be close to the exciton recombination zone, because the diffusion length of singlet excitons is far below that of triplet excitons. ${ }^{1,11-13}$ Compared with the triplet level of yellow or green phosphorescent dopants, fluorescent materials such as p-bis (p-N,N-diphenyl-aminostyryl) benzene (DSA-ph), 4,4'-bis [2-\{4-(N,N-diphenylamino) phenyl \}vinyl]biphenyl (DPAVBi), and 2,2',7,7'-tetrakis(2,2-diphenylvinyl)spiro-9,9'-bifluorene (Spiro-DPVBi) $^{14-18}$ with a lower triplet level inevitably quench the triplet excitons in the exciton recombination zone, giving rise to low external quantum efficiencies (EQEs). In order to alleviate this negative effect, a kind of functional layer called interlayer has been employed in conventional hybrid WOLEDs. ${ }^{8,15,18-25}$

A single hole or electron transport layer can work as a simplest interlayer, ${ }^{8,19-22}$ which is easy to fabricate but cannot simultaneously provide sufficient blue emission by separating the fluorescent EML from the exciton recombination zone. Quenching can also occur in the triplet transfer process

\footnotetext{
${ }^{\text {a) }}$ Author to whom correspondence should be addressed: msdgma@scut.edu.cn and mdg1014@ciac.ac.cn
}

from the exciton recombination zone to the fluorescent emitter with a lower lying non-radiative triplet level.

A mixed interlayer by blending hole and electron transport materials together has been demonstrated to be able to control the position of the exciton recombination zone and manage the singlet and triplet excitons by adjusting the proportion of the two transport materials. ${ }^{8,15,18,23-25}$ The mixed interlayer can effectively prevent triplet quenching in a fluorescent emitter. Nevertheless, the proportions and the thicknesses of such a mixed interlayer have to be precisely controlled, which renders the device fabrication process both cumbersome and difficult to reproduce.

Here, we used a simple planar heterojunction composed of a hole transport material $\mathrm{N}, \mathrm{N}^{\prime}$-diphenyl-N, $\mathrm{N}^{\prime}$-bis(1-naphthylphenyl)-1, $1^{\prime}$-biphenyl-4,4'-diamine (NPB) and an electron transport material bis[2-(2-hydroxyphenyl)-pyridine]beryllium $\left(\mathrm{Bepp}_{2}\right)$ as the interlayer to fabricate high efficiency hybrid WOLEDs. With the structure optimization, the resulting hybrid WOLEDs exhibited maximum efficiencies of $19.3 \%, 57 \mathrm{~cd} \mathrm{~A}^{-1}$, and $661 \mathrm{~m} \mathrm{~W}^{-1}$ and retained $18.9 \%$, $55.9 \mathrm{~cd} \mathrm{~A}^{-1}$, and $60 \mathrm{~lm} \mathrm{~W}^{-1}$ at $1000 \mathrm{~cd} \mathrm{~m}^{-2}$, respectively, without any outcoupling structures. Based on further investigations, such a high performance can be ascribed to the positive effect of the planar heterojunction on the different excited states, owing to the desirable singlet and triplet levels of NPB and Bepp 2 .

We used a blue fluorescent material DSA-ph and an orange phosphorescent material iridium bis(4-phenylthieno $[3,2$-c $]$ pyridinato- $\left.\mathrm{N}, \mathrm{C} 2^{\prime}\right)$ acetylacetonate (PO-01) to fabricate a series of color-complementary hybrid WOLEDs. NPB 


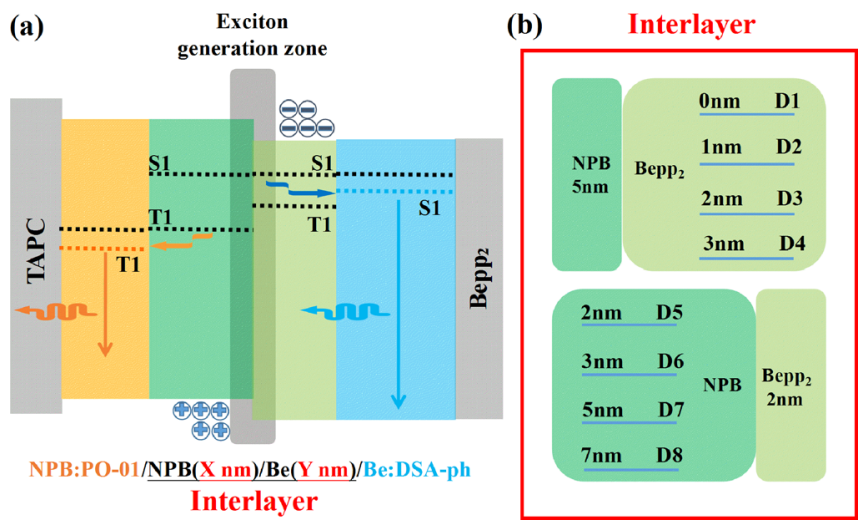

FIG. 1. (a) Schematic diagram of the structure with energy levels and electroluminescence (EL) processes of the resulting hybrid WOLEDs. (b) Detailed structure parameters of the planar heterojunction interlayer in the hybrid WOLEDs.

doped with 15 wt. $\% \mathrm{MoO}_{3}$ and $\mathrm{Bepp}_{2}$ doped with 3.0 wt. \% $\mathrm{Li}_{2} \mathrm{CO}_{3}$ were used as the hole transport layer and electron transport layer, respectively, to improve the device performance. $10 \mathrm{~nm}$-thick 4,4'-cyclohexylidenebis [N, N-bis(p-tolyl) aniline] (TAPC) and Bepp 2 served as electron and hole blocking layers, respectively, to confine carriers and excitons. NPB doped with 5 wt. \% PO-01 and Bepp 2 doped with 2.5 wt. \% DSA-ph acted as the orange phosphorescent EML and blue fluorescent EML, respectively. The planar heterojunction consisting of NPB and $\mathrm{Bepp}_{2}$ layers between two EMLs was used as the interlayer. The final structure of the hybrid WOLEDs is as follows: ITO/ $\mathrm{MoO}_{3}(10 \mathrm{~nm}) /$ NPB: $15 \% \quad \mathrm{MoO}_{3} \quad(50 \mathrm{~nm}) / \mathrm{TAPC}(10 \mathrm{~nm}) / \mathrm{NPB}: 5 \% \quad \mathrm{PO}-01$ $(5 \mathrm{~nm}) / \mathrm{NPB} \quad(\mathrm{x} \quad \mathrm{nm}) / \mathrm{Bepp}_{2} \quad(\mathrm{y} \quad \mathrm{nm}) / \mathrm{Bepp}_{2}: 2.5 \%$ DSA-ph $(5 \mathrm{~nm}) / \mathrm{Bepp}_{2} \quad(10 \mathrm{~nm}) / \mathrm{Bepp}_{2}: 3 \% \quad \mathrm{Li}_{2} \mathrm{CO}_{3}(35 \mathrm{~nm}) / \mathrm{Li}_{2} \mathrm{CO}_{3}$ $(1 \mathrm{~nm}) / \mathrm{Al}$.

As shown in Fig. 1(a), the exciton recombination zone in the series of the hybrid white devices should locate at the interface between NPB and $\mathrm{Bepp}_{2}$ layers due to the high hole and electron mobilities of NPB and $\mathrm{Bepp}_{2}$, respectively. ${ }^{26,27}$ The triplet energy level of DAS-ph $\left(\mathrm{T}_{1} \approx 2.0 \mathrm{eV}\right)$ is lower than that of most of the phosphorescent materials, ${ }^{7,28}$ and it can play the role of the efficient quenching site for triplet excitons in PO-01 molecules. In order to mitigate the aforementioned issue, we introduced a planar heterojunction interlayer composed of a NPB layer and a Bepp 2 layer between the blue fluorescent EML and the orange phosphorescent EML. By considering the fact that the triplet level of $\mathrm{Bepp}_{2}(2.6 \mathrm{eV})$ is higher than that of NPB $(2.3 \mathrm{eV}),{ }^{29-31}$ most triplet excitons can be blocked in the NPB layer and finally transfer from NPB to the orange phosphorescent layer for radiative decay. Moreover, the singlet energy levels of NPB and $\mathrm{Bepp}_{2}$ are approximately equal. ${ }^{32}$ Singlet excitons at the interface between NPB and Bepp 2 can diffuse to both NPB and $\mathrm{Bepp}_{2}$ sides and be used by dopants via Förster energy transfer. Therefore, we can use this kind of interlayer to separate the fluorescent layer and the phosphorescent layer and manipulate the singlet and the triplet excitons efficiently.

To illustrate the effect of the interlayer, the devices with different thicknesses of NPB and Bepp 2 layers have been studied. As shown in Fig. 1(b), we first fixed the thickness of the NPB layer at $5 \mathrm{~nm}$ and varied the thicknesses of Bepp 2 layers from 0 to $3 \mathrm{~nm}$ named D1 to D4. Figures 2(a) and 2(b) show the current density-luminance-voltage $(J-L-V)$ characteristics and EL efficiencies, respectively. It can be seen that all the turn-on voltages of the four devices are about $2.6 \mathrm{~V}$ in Fig. 2(a), meaning that the minor thickness change of electron transport layer Bepp 2 in the planar heterojunction does not affect the electrical property of the fabricated devices, at least in this range of $0-3 \mathrm{~nm}$ owing to the high electron mobility of Bepp 2 .

According to the $\mathrm{CE}$ and $\mathrm{PE}$ versus luminance shown in Fig. 2(b), D3 and D4 with $2 \mathrm{~nm}$ and $3 \mathrm{~nm} \mathrm{Bepp} 2$ exhibit the maximum $\mathrm{CE}$ and $\mathrm{PE}$ of $51.6 \mathrm{~cd} \mathrm{~A}^{-1}$ and $60.4 \mathrm{~lm} \mathrm{~W}^{-1}$ and $51.8 \mathrm{~cd} \mathrm{~A}^{-1}$ and $59.9 \mathrm{~lm} \mathrm{~W}^{-1}$, respectively. At the luminance of $1000 \mathrm{cdm}^{-2}$, the $\mathrm{PE}$ values are still as high as $51.5 \mathrm{~lm} \mathrm{~W}^{-1}$ and $51.0 \mathrm{~lm} \mathrm{~W}^{-1}$. In contrast to D3 and D4, the CEs at the luminance of $1000 \mathrm{~cd} \mathrm{~m}^{-2}$ are only $18.6 \mathrm{~cd} \mathrm{~A}^{-1}$ and $39.4 \mathrm{~cd} \mathrm{~A}^{-1}$ for D1 and D2 with 0 and $1 \mathrm{~nm} \mathrm{Bepp} 2$ as the interlayer. The maximum EQE values of four devices are $10.5 \%, 13.8 \%, 17.9 \%$, and $17.8 \%$, respectively [Fig. S1(a) and supplementary material, Table S1]. It is clearly shown that the thickness of Bepp 2 is the critical factor for achieving high performance devices. Although $\mathrm{Bepp}_{2}$ has a high triplet energy level about $2.6 \mathrm{eV}$, the device with the thickness of the $\mathrm{Bepp}_{2}<2 \mathrm{~nm}$ cannot prevent the triplet excitons transferring from NPB to DSA-ph via Dexter energy transfer, which can lead to triplet quenching in the blue fluorescent EML. With the increasing thickness of Bepp 2 , the blocking effect of Bepp $p_{2}$ turns to be more obvious. When the thickness of $\mathrm{Bepp}_{2} \geq 2 \mathrm{~nm}$, it can keep most of the triplet excitons staying in NPB and prevent the triplet quenching in the fluorescent EML. The triplet excitons can diffuse to orange phosphorescent EML and be used by PO-01 molecules via Dexter energy transfer.

All devices exhibit high EL spectral stability with the luminance increasing from $1000 \mathrm{~cd} \mathrm{~m}^{-2}$ to $10000 \mathrm{~cd} \mathrm{~m}^{-2}$ (supplementary material, Fig. S2). From D1 to D4, the Commission Internationale del'Éclairage $\left(\mathrm{CIE}_{\mathrm{x}, \mathrm{y}}\right)$ coordinates at the luminance of $1000 \mathrm{~cd} \mathrm{~m}^{-2}$ are $(0.38,0.43)$,
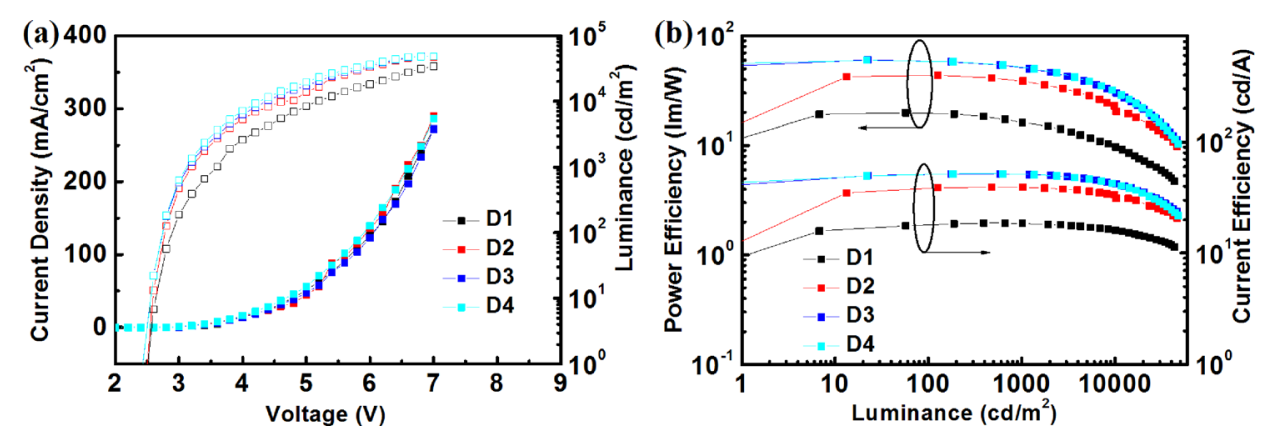

FIG. 2. EL performances of D1 to D4. (a) Current density-luminance-voltage characteristics. (b) Current efficiency (CE) versus luminance and power efficiency $(\mathrm{PE})$ versus luminance. 

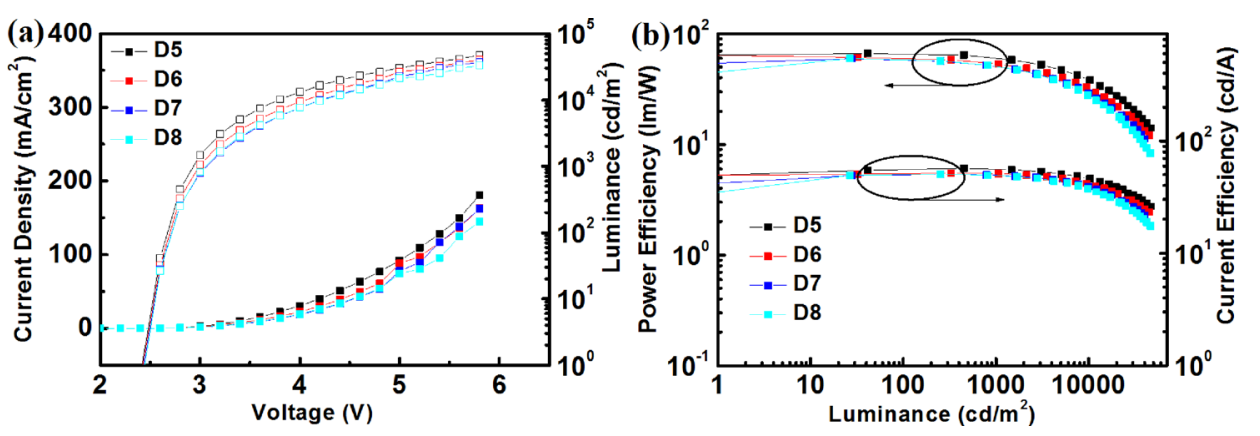

FIG. 3. EL performances of D5 to D8. (a) Current density-luminance-voltage characteristics. (b) CE versus luminance and PE versus luminance.
$(0.42,0.46),(0.44,0.47)$, and $(0.44,0.47)$, respectively. The thickness of Bepp 2 in D1 is $0 \mathrm{~nm}$, and the exciton generation zone is at the interface between the fluorescent layer and the NPB layer; thus. the singlet excitons can directly be harvested by blue fluorescent molecules, leading to the highest blue emission ratio. Meanwhile, with the most triplets quenching, the maximum EQE of D1 is only $10.5 \%$. The blue emission intensity decreases along with the increase in the thickness of Bepp 2 . Since the diffusion length of singlet excitons is relatively short than that of triplet excitons, the singlet exciton density decreases rapidly with the increasing distance from the exciton recombination zone to fluorescent EML, making the blue emission intensity controllable by the thickness of $\mathrm{Bepp}_{2}$.

Then, as shown in Fig. 1(b), we fixed the thickness of Bepp $_{2}$ at $2 \mathrm{~nm}$ and varied the thicknesses of NPB with $2 \mathrm{~nm}$, $3 \mathrm{~nm}, 5 \mathrm{~nm}$, and $7 \mathrm{~nm}$, named D5 to D8, respectively. Figure 3 shows their EL performances, demonstrating that the four devices also have the same turn-on voltages with slightly different current densities. As shown in Fig. 3(b), D5 achieved the maximum $\mathrm{CE}$ and $\mathrm{PE}$ of $57.1 \mathrm{~cd} \mathrm{~A}^{-1}$ and $66.2 \mathrm{~lm} \mathrm{~W}^{-1}$, respectively, and can still retain $60 \mathrm{~lm} \mathrm{~W}^{-1}$ at the luminance of $1000 \mathrm{~cd} \mathrm{~m}^{-2}$. With the structure of D5, very low driving voltages of $3.0 \mathrm{~V}, 3.4 \mathrm{~V}$, and $3.8 \mathrm{~V}$ are obtained for the luminance of $1000 \mathrm{~cd} \mathrm{~m}^{-2}, 5000 \mathrm{~cd} \mathrm{~m}^{-2}$, and $10000 \mathrm{~cd} \mathrm{~m}^{-2}$, respectively.

The thickness of the NPB layer represents the distance between the exciton generation zone and phosphorescent EML. Different from the blocking characteristic of $\mathrm{Bepp}_{2}$ discussed above, the triplet excitons can efficiently transfer from NPB to PO-01 via Dexter energy transfer. The device with a $7 \mathrm{~nm}$-thick NPB layer still shows a high performance. Compared to the devices with the thicknesses of NPB above $3 \mathrm{~nm}$, D5 with $2 \mathrm{~nm}$ NPB obtains the highest EQE of $19.3 \%$, and even at $1000 \mathrm{~cd} \mathrm{~m}^{-2}$, the EQE remains at a high value of 18.9\% [supplementary material, Fig. S1(b) and Table S2]. Considering the fact that the diffusion length of singlet excitons is much shorter than that of triplet excitons, if the distance between the exciton generation zone and phosphorescent EML is beyond the singlet exciton diffusion length, the large amount of singlet excitons would be lost in the NPB layer. When the distance between the exciton generation zone and phosphorescent EML is within the singlet exciton diffusion length, the singlet excitons at the NPB side can be utilized by phosphorescent materials. Moreover, all of the devices maintain stable warm white emission from $1000 \mathrm{~cd} \mathrm{~m}^{-2}$ to $10000 \mathrm{~cd} \mathrm{~m}^{-2}$ (supplementary material, Figs. S3 and S4).
To further demonstrate the effect of the planar heterojunction interlayer, we inserted $0.1 \mathrm{~nm}$ DSA-ph and PO-01 sensitive layers into the different positions in the NPB/Bepp 2 heterojunction interface to detect the singlet and triplet exciton densities. There is no obvious holes trapped by PO-01 molecules in the NPB layer, and both DSA-ph and PO-01 molecules have a slight effect on the electron transfer in the $\mathrm{Bepp}_{2}$ matrix (supplementary material, Fig. S5).

Therefore, we fabricated the devices with a uniform structure of $\mathrm{ITO} / \mathrm{MoO}_{3}(10 \mathrm{~nm}) / \mathrm{NPB}: 15 \% \mathrm{MoO}_{3} \quad(50 \mathrm{~nm}) / \mathrm{TAPC}$ $(10 \mathrm{~nm}) / \mathrm{NPB} \quad(10 \mathrm{~nm}) / \mathrm{Bepp}_{2} \quad(15 \mathrm{~nm}) / \mathrm{Bepp}_{2}: \quad 3 \% \mathrm{Li}_{2} \mathrm{CO}_{3}$ $(35 \mathrm{~nm}) / \mathrm{Li}_{2} \mathrm{CO}_{3}(1 \mathrm{~nm}) / \mathrm{Al}$.

As shown in Fig. 4(a), for the four devices named T1-T4, the distance between the DSA-ph sensor and the interface of NPB/Bepp 2 is $0 \mathrm{~nm}, 1 \mathrm{~nm}, 2 \mathrm{~nm}$, and $3 \mathrm{~nm}$, respectively.

The EL intensities of four devices at the same voltage $(4.0 \mathrm{~V})$ are shown in Fig. 4(b). Since DSA-ph can only utilize the singlet excitons, the EL intensities should correspond to the singlet exciton densities at different positions. The maximum intensity of blue emission is obtained in T1, implying that the density of singlet excitons at the interface position is the highest. With the increasing distance of the sensor away from the interface in $\mathrm{Bepp}_{2}$, the EL intensity reduces gradually. We also obtained roughly half of the maximum intensity when the distance increases to $2 \mathrm{~nm}$, proving the efficient energy transfer of the singlet excitons from the exciton generation zone to fluorescent EML.

(a)

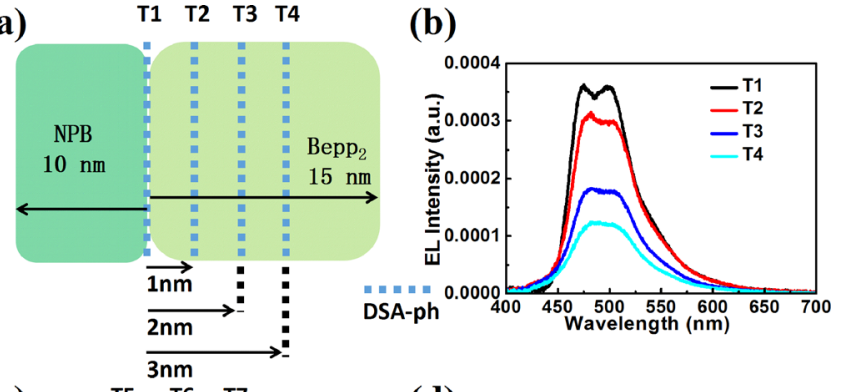

(c)

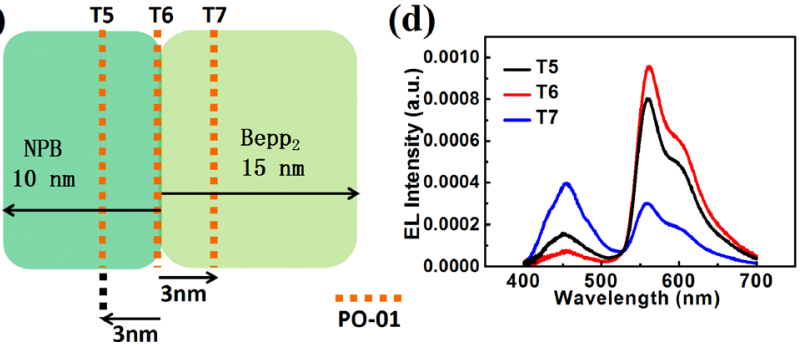

FIG. 4. Positions of DSA-ph and PO-01 sensors and the corresponding EL intensities at $4.0 \mathrm{~V}$. 
In Fig. 4(c), we inserted a $0.1 \mathrm{~nm}$ PO-01 sensitive layer at three different positions in the interlayer to study the exciton distribution, named T5 to T7. The EL intensities of three devices at $4.0 \mathrm{~V}$ can be seen in Fig. 4(d), the maximum EL intensity of orange emission is achieved in $\mathrm{T} 6$, and $\mathrm{T} 5$ exhibits a slightly lower EL intensity of orange emission but much higher than that of T7. This indicates that the triplet excitons can be efficiently transferred in NPB whereas be strongly blocked by Bepp ${ }_{2}$.

We used a planar heterojunction of $\mathrm{NPB} / \mathrm{Bepp}_{2}$ as the interlayer located between fluorescent blue EML and phosphorescent orange EML to fabricate the high efficiency hybrid WOLEDs. With the desirable singlet and triplet energy levels of NPB and Bepp 2 , the singlet and triplet excitons can be separated at the interface between NPB and $\mathrm{Bepp}_{2}$; thus, the quenching caused by the triplet of the fluorescent material can be alleviated effectively. The resulting hybrid OLEDs achieved a stable warm white emission and a high performance of $19.3 \%, 57 \mathrm{~cd} \mathrm{~A}^{-1}$, and $66 \mathrm{~lm} \mathrm{~W}^{-1}$. At the luminance of $1000 \mathrm{~cd} \mathrm{~m}^{2}$, they still retain $18.9 \%, 55.9 \mathrm{~cd}$ $\mathrm{A}^{-1}$, and $60 \mathrm{~lm} \mathrm{~W} \mathrm{~W}^{-1}$, respectively. Our results offer a simple route to realize high performance hybrid WOLEDs.

See supplementary material for the experimental method.

The authors gratefully acknowledge the National Key Research and Development Plan of China (2016YFB0400700), the National Natural Science Foundation of China (Grant Nos. 51333007, 51573180, 91433201, and 11661131001), and Guangzhou Science and Technology Plan Project (201707020040) for the support of this research. Professor D. G. Ma also appreciates the great support from Distinguished Scientist Fellowship Program (DSFP), King Saud University.

${ }^{1}$ Y. Sun, N. C. Giebink, H. Kanno, B. Ma, M. E. Thompson, and S. R. Forrest, Nature 440, 908 (2006).

${ }^{2}$ G. Schwartz, M. Pfeiffer, S. Reineke, K. Walzer, and K. Leo, Adv. Mater. 19, 3672 (2007).

${ }^{3}$ N. Sun, Q. Wang, Y. Zhao, Y. Chen, D. Yang, F. Zhao, J. Chen, and D. Ma, Adv. Mater. 26, 1617 (2014).

${ }^{4}$ C.-J. Zheng, J. Wang, J. Ye, M.-F. Lo, X.-K. Liu, M.-K. Fung, X.-H. Zhang, and C.-S. Lee, Adv. Mater. 25, 2205 (2013).
${ }^{5}$ B.-Q. Liu, L. Wang, D.-Y. Gao, J.-H. Zou, H.-L. Ning, J.-B. Peng, and Y. Cao, Light: Sci. Appl. 5, e16137 (2016).

${ }^{6}$ G. Schwartz, S. Reineke, T. C. Rosenow, K. Walzer, and K. Leo, Adv. Funct. Mater. 19, 1319 (2009).

${ }^{7}$ J. Chen, F. Zhao, and D. Ma, Mater. Today 17, 175 (2014).

${ }^{8}$ B. Liu, H. Nie, X. Zhou, S. Hu, D. Luo, D. Gao, J. Zou, M. Xu, L. Wang, Z. Zhao, A. Qin, J. Peng, H. Ning, Y. Cao, and B. Z. Tang, Adv. Funct. Mater. 26, 776 (2016).

${ }^{9}$ J. Li, L. Xu, S. Kim, and A. A. Shestopalov, J. Mater. Chem. C 4, 4155 (2016).

${ }^{10}$ J. Li, L. Xu, C. W. Tang, and A. A. Shestopalov, ACS Appl. Mater. Interface 8, 16809 (2016).

${ }^{11}$ S. Hofmann, T. C. Rosenow, M. C. Gather, B. Lüssem, and K. Leo, Phys. Rev. B 85, 245209 (2012).

${ }^{12}$ J. Wünsche, S. Reineke, B. Lüssem, and K. Leo, Phys. Rev. B 81, 245201 (2010).

${ }^{13}$ J. Ye, C.-J. Zheng, X.-M. Ou, X.-H. Zhang, M.-K. Fung, and C.-S. Lee, Adv. Mater. 24, 3410 (2012).

${ }^{14}$ H. I. Baek and C. H. Lee, J. Phys. D: Appl. Phys. 41, 105101 (2008).

${ }^{15}$ G. Schwartz, T.-H. Ke, C.-C. Wu, K. Walzer, and K. Leo, Appl. Phys. Lett. 93, 073304 (2008).

${ }^{16}$ T. Xu, M. Yang, J. Liu, X. Wu, I. Murtaza, G. He, and H. Meng, Org. Electron. 37, 93 (2016).

${ }^{17}$ Y. Liu, L.-S. Cui, M.-F. Xu, X.-B. Shi, D.-Y. Zhou, Z.-K. Wang, Z.-Q. Jiang, and L.-S. Liao, J. Mater. Chem. C 2, 2488 (2014).

${ }^{18}$ G. Schwartz, K. Fehse, M. Pfeiffer, K. Walzer, and K. Leo, Appl. Phys. Lett. 89, 083509 (2006).

${ }^{19}$ B. Liu, Z. Xu, J. Zou, H. Tao, M. Xu, D. Gao, L. Lan, L. Wang, H. Ning, and J. Peng, J. Ind. Eng. Chem. 27, 240 (2015).

${ }^{20}$ Q. Wang, C.-L. Ho, Y. Zhao, D. Maa, W.-Y. Wong, and L. Wang, Org. Electron. 11, 238 (2010).

${ }^{21}$ B.-P. Yan, C. C. C. Cheung, S. C. F. Kui, H.-F. Xiang, V. A. L. Roy, S.-J. Xu, and C.-M. Che, Adv. Mater. 19, 3599 (2007).

${ }^{22}$ B. Liu, M. Xu, L. Wang, X. Yan, H. Tao, Y. Su, D. Gao, L. Lan, J. Zou, and J. Peng, Org. Electron. 15, 926 (2014).

${ }^{23}$ Y. Liu, F. Liang, L.-S. Cui, X.-B. Shi, Z.-K. Wang, and L.-S. Liao, Adv. Opt. Mater. 4, 2051 (2016).

${ }^{24}$ F. Zhao, Z. Zhang, Y. Liu, Y. Dai, J. Chen, and D. Ma, Org. Electron. 13, 1049 (2012).

${ }^{25}$ G. Schwartz, S. Reineke, K. Walzer, and K. Leo, Appl. Phys. Lett. 92, 053311 (2008).

${ }^{26}$ Y. Li, Y. Liu, W. Bu, D. Lu, Y. Wu, and Y. Wang, Chem. Mater. 12, 2672 (2000).

${ }^{27}$ T.-Y. Chu and O.-K. Song, Appl. Phys. Lett. 90, 203512 (2007).

${ }^{28}$ T. Zheng and W. C. H. Choy, Adv. Funct. Mater. 20, 648 (2010).

${ }^{29}$ S. H. Kim, J. Jang, and J. Y. Lee, Appl. Phys. Lett. 90, 223505 (2007).

${ }^{30}$ M. Du, Y. Feng, D. Zhu, T. Peng, Y. Liu, Y. Wang, and M. R. Bryce, Adv. Mater. 28, 5963 (2016).

${ }^{31}$ T. Peng, Y. Yang, H. Bi, Y. Liu, Z. Houb, and Y. Wang, J. Mater. Chem. 21, 3551 (2011).

${ }^{32}$ F. Zhao, Y. Wei, H. Xu, D. Chen, T. Ahamad, S. Alshehri, Q. Pei, and D. Ma, Mater. Horiz. 4, 641 (2017). 